durchflusszytometrische Methode, mit deren Hilfe sich die Allergen-IgE-Bindung an den niedrigaffinen IgE-Rezeptor FceRII (CD23) auf den B-Zellen nachweisen lässt. Mit dieser Methode wurden die Seren von Patienten mit einer schweren saisonalen Rhinitis, u. a. aufgrund einer Allergie gegen Phleum pratense, untersucht - und zwar jeweils zum Höhepunkt der Pollenflugzeit vor und nach einer einjährigen subkutanen Immuntherapie. Zehn Patienten hatten über das eine Jahr Verum-, acht Patienten Plazebo-Injektionen erhalten.

Bei allen Patienten unter Verum wurde die Allergen-IgE-Bindung an die B-Zellen unterdrückt ( $p=0,02$ vs. Pla-
zebo-Patienten). Auch die anschließende Allergenpräsentation durch die BZellen unterblieb. Dieser blockierende Effekt war auf die Anwesenheit Allergen-spezifischer IgG-Antikörper zurückzuführen, deren Serumspiegel durch die Verum-Behandlung deutlich angestiegen war. Das neu gebildete IgG war Allergen-spezifisch: Bei Patienten, die gegen Gräserpollen hyposensibilisiert worden waren, gleichzeitig aber auch noch unter Birkenpollenallergie litten, wurde die BirkenpollenallergenIgE-Bindung an die B-Zellen nicht unterbunden.

Durch welchen Mechanismus die „blockierenden“ Antikörper gebildet werden, ist unklar, ebenso wie das $\mathrm{Zu}$ sammenspiel mit anderen Faktoren, die bei der SIT eine Rolle spielen.

Fazit: Eine Hyposensibilisierung induziert die Bildung Allergen-spezifischer IgG-Antikörper, die die IgE-vermittelte Allergenbindung an B-Zellen blockieren und so die Allergenpräsentation an die T-Zellen verhindern. Möglicherweise ist dies ein zentraler Wirkmechanismus der SIT.

Wachholz PA et al. Inhibition of allergenIgE binding to B cells by IgG antibodies after grass pollen immunotherapy. J Allergy Clin Immunol 2003; 112: 915-22

\title{
Studien zwischen Fakt und Fiktion
}

\section{Nicht immer sind Ergebnisse von klinischen Therapiestudien, die sich an Kriterien der evidenzbasierten Medizin orientieren, auch im Praxisalltag Wegweiser für Therapieentscheidungen.}

$E_{\text {ter }}^{\text {in }}$ n entscheidender limitierender Faktor bei der Übertragbarkeit klinischer Studienergebnisse in den Praxisalltag sind die strengen Ausschlusskriterien klinischer Studien. Sie sollen Interaktionen und Störfaktoren minimieren, die im normalen Patientengut aber gang und gäbe sind (vgl. Kasten rechts). Schätzungen gehen davon aus, dass dadurch nur rund 15\% aller Asthmapatienten und rund $30 \%$ aller Rhinitispatienten für klinische Studien in Frage kommen.

Auch ist die Compliance in den meist nur über einen relativ kurzen Zeitraum durchgeführten klinischen Studien naturgemäß besser als unter Langzeitbedingungen. So ergab eine Langzeitstudie über bis zu 140 Wochen zunächst eine Überlegenheit von inhalativem Beclometason gegenüber oralem Montelukast beim Studienendpunkt $\mathrm{FEV}_{1}$. Doch fielen im Verlauf der Untersuchung die $\mathrm{FEV}_{1}$ - Werte der Patienten unter der inhalativen Therapie wieder, während sie bei den Patienten unter dem oralen Leukotrien-Rezeptorantagonisten konstant blieben. Hier dürften Compliancefaktoren eine wichtige Rolle gespielt haben.
Ein weiterer Kritikpunkt sind primäre Studienendpunkte wie $\mathrm{FEV}_{1}$, krankheitsspezifische Lebensqualität oder der Gesamtscore nasaler Symptome, die fast zum Standard bei Studien in der Allergologie geworden sind. Für die Studien sind diese Parameter mit ihrer einfachen Quantifizierbarkeit ideal, spiegeln allerdings die Erwartungen an ein gutes, an die Patientenbedürfnisse angepasstes Asthma- oder Rhinitismedikament nur partiell wider.

Die in den Studienergebnissen angegeben Durchschnittswerte sind in der Praxis beim einzelnen Patienten nicht zu erwarten. Die Ergebnisse von Subgruppenanalysen zeigen häufig, dass bestimmte Patienten besonders gut, andere dagegen nur schlecht auf ein Medikament ansprechen - eine Beobachtung, die der Arzt auch bei seinen Patienten machen muss.

Und schließlich erhalten die an Studien beteiligten Zentren häufig finanzielle Unterstützung von der Pharmaindustrie. Dies führt möglicherweise zu nicht objektiven Ergebnissen bzw. zu einer Tendenz, positive Ergebnisse zu publizieren, negative dagegen eher nicht zu veröffentlichen.
Typische Ausschlusskriterien bei Asthma- und Rhinitisstudien

— Rauchen in der Anamnese

— Vorliegen von Begleiterkrankungen wie Asthma oder Sinusitis (Rhinitisstudien)

_ FEV der Patienten ist zu hoch oder zu niedrig (Asthmastudien).

Warum Patienten nicht an Studien teilnehmen

_ Aktuelle Medikation ist effektiv.

- Patienten sind nicht bereit, bestimmte im Studienprotokoll nicht vorgesehene Medikamente abzusetzen.

— Patienten setzen Akutmedikamente zu häufig oder zu selten ein (Asthmastudien).

— Patienten fürchten eine Verschlechterung ihres Zustandes während der Wash-out-Phase (Asthmastudien).

Fazit: Die randomisierte kontrollierte Studie ist zu Recht das Maß aller Dinge bei der klinischen Entscheidungsfindung. Für die Alltagstauglichkeit der Ergebnisse wäre aber zu wünschen, dass der plazebokontrollierten Phase eine offene Langzeitphase folgt.

Storms W. Clinical trials: are these you patients? J Allergy Clin Immunol 2003; 112: S107-11 